\title{
Workshop on the Serological Testing Algorithm FOR RECENT HIV SEROCONVERSION (STARHS) AND HIV InCIDENCE ESTIMATES, StOCKHOLM, 11-12 MarCh 2008
}

Editorial team (eurosurveillance@ecdc.europa.eu) ${ }^{1}$

1. Eurosurveillance, European Centre for Disease Prevention and Control, Stockholm, Sweden

The recent development of serological assays for human immunodeficiency virus (HIV) that are able to distinguish recent from long-standing infection has generated an important tool for HIV surveillance. In the European Union (EU), a number of different serological assays are being used, and there is the danger that that HIV incidence estimates in different countries, or even within a country, may not be comparable.

The former EU-funded project EURO HIV (http://ec.europa. eu/health/ph_projects/2004/action2/action2_2004_13_en.htm) included a work package on the investigation of several serological assays for recent HIV infection. It investigated the transferability of these tests, their comparative performance and their application in estimating HIV incidence in selected populations. Ten EU Member States contributed to this work.

The HIV experts (both epidemiologists and virologists) came together at a workshop held on 11 and 12 March 2008 at the European Centre for Disease Prevention and Control (ECDC) in Stockholm, Sweden, to discuss different approaches of the Serological Testing Algorithm for Recent HIV Seroconversion (STARHS) and their use for estimating HIV incidences.

EU-wide comparison of HIV serological methods

The EURO HIV 'work package 7' was presented by John Parry (Health Protection Agency (HPA), London, United Kingdom (UK)). It aimed to increase networking and cooperation between reference laboratories with the goal of harmonising surveillance methods in the EU in order to obtain comparable data across countries. It investigated the use of the following serological assays:

- 'Detuned' enzyme immuno-assays (EIA) (modified commercial assays; bioMerieux Vironostika and Abbot HIV AB 3A11)

- BED-CEIA: an antibody capture EIA measuring the IgG proportion (commercial; Calypte Biomedical)

- AxSYM: an antibody avidity assay (modified commercial; Abbot)

- IDE-V3: EIA targeting two antigens at the same time (in-house assay; produced by Francis Barin)

Gary Murphy (HPA, London, UK) gave an overview of the existing serological assays that are able to distinguish between recent and long-standing HIV infection, pointing out the advantages and drawbacks of each method and identifying desirable criteria for an ideal assay. A more detailed description of the individual assays can be found in the article by Murphy and Parry in this issue.
The list of desirable characteristics for a STARHS assay includes a well defined, preferably long, window period, consistent discrimination between recent and long-standing infection, and accurate results for different cut-off values. The result should be independent of factors such as virus subtype, mode of transmission, opportunistic infections, pregnancy, and age, sex, race and therapy status of the patient. On the operational side, cost, availability, equipment requirements, ease of handling and storage, and the suitability for small volumes and different types of samples need to be taken into account, and the assay should ideally not depend on a single company. Moreover, a programme to standardise and control the performance of the assay needs to be in place.

It was concluded that no single assay at present fulfils all the desired characteristics.

All four STARHS methods were compared at the HPA Centre for Infections ( $\mathrm{CfI}$ ), London, though some were in use in other laboratories so that limited further comparisons using the same specimen panels were possible. The panel comprised 374 well characterised samples from England ( $\mathrm{Cfl}$, London) and France (Université François Rabelais (UFR), Tours), as well as seven panels of around 200 samples from new HIV diagnoses that had been collected in England, Finland, Germany, Italy, the Netherlands, Portugal and Spain, a total of 1,736 eligible specimens. The results of the comparison are available in the final EURO HIV reports (available at: http://www.hpa.org.uk/webw/ HPAweb\&HPAwebStandard/HPAweb_C/1195733851609? $p=120$ 0660013708).

The intra-laboratory reproducibility was found to be satisfactory, with a reasonable correlation between original and repeat test results for the BED, Detuned, Avidity and IDE-V3 assays used at the $\mathrm{CfI}$, and the IDE-V3 assay used at the UFR. However, certain issues were raised such as the need to define a window-period for the AxSYM and IDE-V3 tests, the need to set up a confirmatory test algorithm, particularly for specimens that give results in a critical range around the threshold value, and the evidence that a minority of patients may never develop an immune response sufficient to convert to a long-standing status in some assays. It was also seen as important to take into account the different factors that may bias the results, for instance anti-retroviral treatment, virus subtype, and disease stage. 
There was some inter-laboratory variability that the participants thought was due to equipment calibration or maintenance issues and differences in the production lots purchased from the companies. They emphasised the importance of experience and training regarding the equipment and of suitable calibrators and controls, which are still to be developed. The lack of appropriate external assessment programmes to assure the quality of STARHS testing was seen as one of the greatest barriers to the transfer of any given method between different laboratories. It was suggested that it may be necessary to define a reference laboratory responsible for the development and standardised evaluation of new STARHS methods.

Andre Charlett (HPA, London, UK) presented an assessment of whether there was agreement between the four STARHS assays in the identification of recent versus long-standing HIV infection when using different window periods. The classification of the majority of specimens was consistent, but there were also intolerable inconsistencies, and none of the assays was found to be suitable for every specimen.

\section{HIV incidence in the EU}

With the laboratory methods still in need of improvement, more uncertainties arise when transferring laboratory data to incidence estimation. Part of 'work package 7' was designed to test the applicability of the STARHS results for HIV incidence estimates in selected subpopulations in different EU Member States. Preliminary results from an HIV incidence estimation in three collaborating countries were presented by Daniela DeAngelis (HPA, London).

The estimates based on data from the four different STARHS assays differed substantially, and it was felt that more discussion will be needed on the interpretation of the results. Three main problems were put forward as possible reasons for the discrepant results: a) the data collection methodology may influence the interpretation of the test results; b) the difficulty of estimating the distribution of the window period, as the estimation procedure involves many assumptions and it might be based on a small panel of seroconverters; and c) misclassification of long-standing infections as recent. Other factors influencing the result include epidemiological data such as the testing pattern, the time since the last negative test and behavioural data.

\section{Ongoing international activities in Europe}

The second day of the meeting began with an overview on other ongoing European programmes focused on HIV incidence estimation. After a short presentation outlining the ECDC laboratory strategy, Valerie Delpech (HPA, London, UK) presented the EUfunded project 'Concerted Action on SeroConversion to AIDS and Death in Europe' (CASCADE), a network of epidemiologists, statisticians, virologists and clinicians from leading HIV institutions in 15 European countries, Australia and Canada that collects lifelong data from local and national cohorts of seroconverters.

CASCADE's current activities include the ascertainment and follow-up of recently infected people in central and eastern Europe. Since most countries do not have the facilities to start a new largescale surveillance project, CASCADE plans site visits to laboratories in order to create the infrastructure and train staff in suitable HIV tests. Whether STARHS methods are appropriate in this context, is being discussed.
The session was concluded with feedback from a recent meeting of the WHO working group on HIV incidence assays, a worldwide initiative to establish best practice in the calibration and evaluation of STARHS methodologies, to study the evidence on the use of these assays and to provide guidance on appropriate approaches to measuring HIV incidence. The next steps of the project foresee supporting the establishment of appropriate specimen panels, the calibration of existing and the development of new assays as well as their application, and the determination of a window period. A statistics working group will advise on how to interpret results and determine incidences. The use of incidence assays for purposes other than incidence estimates is being discussed.

\section{Future objectives}

In a third session the participants discussed, in two working groups, the laboratory and epidemiological aspects of using various STARHS assays, in order to define the next steps regarding the development and implementation of HIV serological assays and regarding incidence modelling in the EU Member States.

The workshop participants agreed that it is advisable to have at least two satisfactory standard STARHS methods established in all laboratories undertaking STARHS testing, in case one test should be temporarily unavailable. ECDC had hoped to conclude this workshop with a recommendation of one or two of these assays and to discuss the feasibility of their implementation in the EU. However, the experts felt that information for such a decision was lacking, and there was a general agreement that it is at present not possible to make such a recommendation. The laboratory experts were of the opinion that in the medium term it was more likely that five or six different assays would be in use across Europe and stressed that quality assessment programmes would be needed for all of them.

It was agreed that once an agreement has been reached on the test(s) to be used, ECDC should coordinate and fund the development of a framework or guideline for the implementation of STARHS for epidemiological use, detailing what epidemiological data are needed, from which populations, and which sampling strategy should be used. In the meantime, more work needs to be done with regards to the estimation of the window period, and a quality assurance and training programme needs to be developed. Further urgent issues for the near future include the development of an EU-specific panel of seroconverter samples for calibration of the assays, the realistic window period estimates, and a deeper analysis of the epidemiological information including validation of the results in different population groups according to the different factors that may bias the results.

It was decided that the WHO global initiative should be followed closely to avoid duplication of work. The overall conclusion was that, while HIV incidence testing may not become part of routine HIV surveillance in the very near future, all efforts regarding test development and epidemiological sampling frame should be targeted to reach this stage as soon as possible in order to improve the understanding of HIV epidemiology in the EU.

This article was published on 4 September 2008.

Citation style for this article: Editorial team. Workshop on the Serological Testing Algorithm for Recent HIV Seroconversion (STARHS) and HIV Incidence Estimates, Algorithm for Recent HIV Seroconversion (STARHS) and HIV Incidence Estimates,
Stockholm, 11-12 March 2008. Euro Surveill. 2008:13(36): pii=18972. Available online: Stockholm, 11-12 March 2008. Euro Surveill. 2008;13(36):pii=18972.
http://www.eurosurveillance.org/ViewArticle.aspx?ArticleId $=18972$ 